\title{
Three-dimensional particle simulation of plasma instabilities and collisionless reconnection in a current sheet
}

\author{
Ritoku Horiuchi and Tetsuya Sato \\ Theory and Computer Simulation Center, National Institute for Fusion Science, Toki 509-52, Japan
}

(Received 22 June 1999; accepted 24 August 1999)

\begin{abstract}
Generation of anomalous resistivity and dynamical development of collisionless reconnection in the vicinity of a magnetically neutral sheet are investigated by means of a three-dimensional particle simulation. For no external driving source, two different types of plasma instabilities are excited in the current layer. The lower hybrid drift instability (LHDI) is observed to grow in the periphery of current layer in an early period, while a drift kink instability (DKI) is triggered at the neutral sheet in a late period as a result of the nonlinear deformation of the current sheet by the LHDI. A reconnection electric field grows at the neutral sheet in accordance with the excitation of the DKI. When an external driving field exists, the convective electric field penetrates into the current layer through the particle kinetic effect and collisionless reconnection is triggered by the convective electric field earlier than the DKI is excited. It is also found that the anisotropic ion distribution is formed through the anomalous ion heating by the DKI. () 1999 American Institute of Physics. [S1070-664X(99)01412-3]
\end{abstract}

\section{INTRODUCTION}

Collisionless reconnection is widely believed to play a key role in energetically active phenomena observed in a high temperature, rarefied (collisionless) plasma such as the solar corona, ${ }^{1}$ the geomagnetic tail, ${ }^{2}$ laboratory plasmas, ${ }^{3-5}$ fusion plasmas, ${ }^{6}$ and so on. Magnetic reconnection can lead to a fast energy conversion from an electromagnetic field to particles as well as a topological change of magnetic field. Magnetic reconnection, however, cannot take place in an ideal magnetohydrodynamic plasma because the magnetic field is frozen in a plasma. That is, a nonideal effect which breaks the frozen-in condition is needed for the excitation of magnetic reconnection. A number of theoretical and simulation studies have disclosed that there exist two types of triggering mechanisms which break the frozen-in condition of the magnetic field and lead to magnetic reconnection in a collisionless plasma. One is due to the wave-particle interaction which is a cause of anomalous resistivity in the current sheet. ${ }^{7-26}$ The other is due to the particle kinetic effect which becomes significant in a particle scale such as an electron collisionless skin depth ${ }^{27}$ and an ion Larmor radius. ${ }^{28-30}$

A variety of plasma instabilities have so far been studied as a candidate of an anomalous resistivity in a current sheet. Most of these studies focus on the ion-acoustic instability, ${ }^{7}$ the whistler instability, ${ }^{8-10}$ and the collisionless tearing instability ${ }^{11-17}$ which propagate in the direction of an equilibrium magnetic field. Winske, ${ }^{18}$ Tanaka, ${ }^{19}$ and Ozaki et $a l^{20}{ }^{20}$ have found from two-dimensional particle simulation that two kinds of plasma instabilities grow in the current layer, i.e., the lower hybrid drift instability (LHDI) ${ }^{13,18,21,22}$ and a low frequency electromagnetic (EM) instability. ${ }^{13,18,23-26}$ The LHDI grows in the periphery of the current layer, but it cannot penetrate into a high beta region in the vicinity of the neutral sheet. Ozaki et al. ${ }^{20}$ have pointed out that a low frequency electromagnetic (EM) instability, which is called the drift-kink instability (DKI), ${ }^{24}$ is excited near the neutral sheet after the saturation of the LHDI and can be a cause of anomalous resistivity at the neutral sheet. $\mathrm{Zhu}^{24}$ has also pointed out that the DKI is driven by the peaked current and propagates in the direction of an equilibrium current. One of the important features of the DKI is that the DKI grows with a fairly large rate when the width of a current layer decreases below the ion Larmor radius, ${ }^{23,26}$ and that the excitation of the DKI is deeply related to the existence of the LHDI. $^{18}$

It is important to examine particle kinetic effects in collisionless reconnection because an electric resistivity was observed to increase anomalously in reconnection experiments $^{3-5}$ when the width of the current sheet approaches the ion Larmor radius. Furthermore, a thin current layer was observed in the geomagnetic tail region, the width of which is comparable to the ion Larmor radius. ${ }^{31}$ By using two-dimensional particle simulation Horiuchi and Sato ${ }^{28,29}$ have examined the particle kinetic effects on collisionless reconnection for the open system which is subject to an external driving field. Collisionless driven reconnection develops in two steps in accordance with the formation of two current layers, i.e., ion current layer in which the ion kinetic effect is dominant, and electron current layer in which the electron kinetic effect is dominant. They have found that the whole dynamical evolution of collisionless reconnection is controlled by the ion dynamics rather than the electron dynamics even if reconnection is triggered by the electron dynamics, and that the saturated reconnection rate is determined by an external driving field. These features are commonly observed in the phenomena triggered by driven magnetic reconnection. ${ }^{32,33}$ The anomalous ion heating takes place through the ambipolar interaction by an electrostatic field generated in the downstream. ${ }^{28,29}$

Which of the two triggering mechanisms is more effec- 


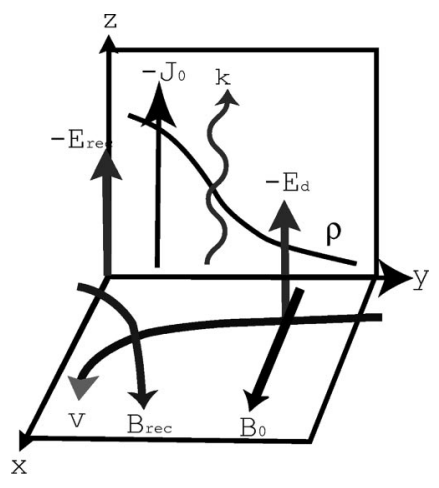

FIG. 1. A schematic diagram of plasma instabilities and collisionless reconnection. Here $\mathbf{B}_{0}, \mathbf{J}_{0}, \mathbf{E}_{\text {rec }}$, and $\mathbf{B}_{\text {rec }}$ stand for an equilibrium magnetic field, an equilibrium current, a reconnection electric field, and a reconnected magnetic field, respectively. The wavy line shows a wave generated through a plasma instability.

tive in the real situation? Or, is the combined mechanism essential in determining the nature of collisionless reconnection? We have carried out two different types of twodimensional particle simulations in relation to the anomalous resistivity and its influence on collisionless reconnection. Figure 1 shows a schematic diagram of two particle simulations. Nonlinear evolution of collisionless reconnection triggered by particle kinetic effects has been examined in the reconnection plane $(x-y$ plane) which is normal to an equilibrium current $\mathbf{J}_{0} \cdot{ }^{28,29}$ On the other hand, the anomalous resistivity, which is originated from the interaction between charged particles carrying the equilibrium current and waves propagating along the equilibrium current (wavy line in Fig. $1)$, has been examined in the simulation domain $(y-z$ plane $)$ which is normal to the reconnection plane. ${ }^{20}$ Suppose that the current layer with a large drift velocity is created as a result of the compression by a convergent plasma flow. Then, the current sheet may become unstable against a plasma instability such as a variety of drift instabilities if the drift velocity exceeds some critical value. The wave propagating in the direction of the average drift motion of charged particles $(z$ direction) is generated through such a wave-particle interaction. A part of the momentum which is carried by charged particles in the current layer is transferred to the wave and carried away from the current layer. This momentum loss suggests that the wave-particle interaction acts as an effective collision. The electric field $\left(\mathbf{E}_{\mathrm{rec}}\right)$ along the equilibrium current $\left(\mathbf{J}_{0}\right)$ is induced at the neutral sheet and accelerates charged particles to compensate the momentum loss. At the same time magnetic reconnection takes place in the presence of the electric field. The reconnected magnetic flux $\left(\mathbf{B}_{\mathrm{rec}}\right)$ is carried away towards the downstream by the generated divergent plasma flow. Consequently, the spatial structure appears in the direction of an equilibrium magnetic field $\left(\mathbf{B}_{0}\right)$. Thus, three-dimensional treatment is needed in order to investigate the dynamical evolution of the system in which two triggering mechanisms become active simultaneously and interact mutually.

The purpose of this article is to demonstrate a threedimensional particle simulation of collisionless reconnection in the presence of an external driving field and to clarify the relationship between two mechanisms. The numerical scheme and the simulation model are described in Sec. II. We will discuss the anomalous resistivity associated with plasma instabilities in the absence of an external driving field in Sec. III and the effect of an external driving field on collisionless reconnection in Sec. IV. Finally, a brief summary is given in Sec. V.

\section{SIMULATION MODEL}

Let us consider a three-dimensional open system which evolves dynamically in the presence of an external driving flow. The simulation is carried out by using threedimensional EM particle codes. ${ }^{34}$ The basic equations to be solved are the equations of motion

$$
\begin{aligned}
& \frac{d\left(\gamma_{j} \mathbf{v}_{j}\right)}{d t}=\frac{q_{j}}{m_{j}}\left[\mathbf{E}+\frac{\mathbf{v}_{j}}{c} \times \mathbf{B}\right], \\
& \frac{d \mathbf{x}_{j}}{d t}=\mathbf{v}_{j},
\end{aligned}
$$

and the Maxwell equations

$$
\begin{aligned}
& \frac{1 \partial \mathbf{B}}{c \partial t}=-\nabla \times \mathbf{E}, \\
& \frac{1 \partial \mathbf{E}}{c \partial t}=\nabla \times \mathbf{B}-4 \pi \mathbf{j}, \\
& \nabla \cdot \mathbf{B}=0, \\
& \nabla \cdot \mathbf{E}=4 \pi \rho,
\end{aligned}
$$

where $\mathbf{x}_{j}(t), \mathbf{v}_{j}(t), m_{j}$, and $q_{j}$ are the position, the velocity, the rest mass, and the charge of the $j$ th particle, and the relativistic $\gamma$-factor of the $j$ th particle is defined by

$$
\gamma_{j}=1 / \sqrt{1-\left(\mathbf{v}_{j} \cdot \mathbf{v}_{j}\right) / c^{2}} .
$$

The current density $\mathbf{j}(\mathbf{x}, \mathbf{t})$ and the charge density $\rho(\mathbf{x}, t)$ are obtained by summing over all the particles, namely,

$$
\begin{aligned}
& \mathbf{j}(\mathbf{x}, \mathbf{t})=\sum_{j=1}^{N} \frac{q_{j} \mathbf{v}_{j}(t)}{c} S\left(\mathbf{x}-\mathbf{x}_{j}(t)\right), \\
& \rho(\mathbf{x}, t)=\sum_{j=1}^{N} q_{j} S\left(\mathbf{x}-\mathbf{x}_{j}(t)\right),
\end{aligned}
$$

where $N$ is the total number of particles and $S(\mathbf{x})$ is the form function of particles. ${ }^{28,34}$

As an initial condition we adopt a one-dimensional equilibrium with the Harris-type antiparallel magnetic configuration as

$$
\begin{aligned}
& \mathbf{B}(y)=\left(B_{x}(y), 0,0\right), \\
& B_{x}(y)=B_{0} \tanh (y / L), \\
& P(y)=B_{0}^{2} / 8 \pi \operatorname{sech}^{2}(y / L),
\end{aligned}
$$

where $B_{0}$ is a constant and $L$ is the scale height along the $y$-axis. There is a magnetically neutral sheet at $y=0$ in the initial equilibrium. The initial particle distribution is assumed to be a shifted Maxwellian with a spatially constant 
TABLE I. Simulation parameters, where the second column $\left(n_{x} \times n_{y} \times n_{z}\right)$ stands for the number of spatial grids and $N_{\mathrm{pt}}$ is the total number of particles.

\begin{tabular}{cccccc}
\hline \hline Case & $n_{x} \times n_{y} \times n_{z}$ & $N_{\mathrm{pt}}$ & $E_{0}$ & $\gamma_{\mathrm{LHDI}} / \omega_{c e}$ & $\gamma_{\mathrm{DKI}} / \omega_{c e}$ \\
\hline $\mathrm{A}$ & $32 \times 128 \times 64$ & $6 \times 10^{6}$ & 0.0 & 0.0079 & 0.0035 \\
$\mathrm{~B}$ & $64 \times 64 \times 64$ & $6 \times 10^{6}$ & 0.0 & 0.0046 & 0.0040 \\
$\mathrm{C}$ & $64 \times 64 \times 64$ & $1.2 \times 10^{7}$ & -0.02 & 0.0118 & 0.0096 \\
$\mathrm{D}$ & $32 \times 64 \times 64$ & $6 \times 10^{6}$ & -0.02 & 0.0145 & 0.0073 \\
\hline \hline
\end{tabular}

temperature and the average particle velocity, which is equal to the diamagnetic drift velocity. Because both an ion and an electron are loaded at the same spatial position, there is no electric field in the initial profile.

It is assumed that physical quantities are periodic at the boundary of the $x$-axis $\left(x= \pm x_{b}\right)$ and that of the $z$-axis $(z$ $\left.= \pm z_{b}\right)$, and a driving electric field $\mathbf{E}_{d}(x, t)$ $=\left(0,0, E_{d z}(x, t)\right)$ exists at the boundary of the y-axis $\left(y= \pm y_{b}\right)$ in order to supply the plasma with the $\mathbf{E} \times \mathbf{B}$ drift velocity into the simulation domain. The profile of $E_{d z}(x, t)$ is given by

$$
E_{d z}(x, t)=E_{*}(t)\left\{\epsilon_{f}+\left(1-\epsilon_{f}\right)(\cos (\phi(x))+1) / 2\right\},
$$

where

$$
\begin{aligned}
& E_{*}(t)=\left\{\begin{array}{l}
E_{0}\left(1-\cos \left(\pi t / t_{1}\right)\right) / 2 \text { if } t \leqslant t_{1}, \\
E_{0} \text { if } t>t_{1},
\end{array}\right. \\
& \phi(x)= \begin{cases}-\pi & \text { if } x<-x_{b 1}, \\
\pi x / x_{b 1} & \text { if } x_{b 1} \geqslant x \geqslant-x_{b 1}, \\
\pi & \text { if } x>x_{b 1}\end{cases}
\end{aligned}
$$

$E_{0}$ is a constant, $\epsilon_{f}=0.7, x_{b 1}=x_{b}, t_{1}=65 \omega_{p e}\left(\omega_{p e}\right.$ : a typical electron plasma frequency). In order to avoid the excitation of unphysical waves in the vicinity of the input boundary an artificial damping zone is embedded near the input boundaries $\left(y_{b}-|y| \leqslant 5 \lambda_{d}, \lambda_{d}\right.$; an electron Debye length). We carry out four simulation runs with different parameters which are shown in Table I. The other important parameters are as follows: the ratio of ion to electron temperature is $T_{i} / T_{e}=2$, the ion Larmor radius associated with a magnetic field outside the current layer is $\rho_{i}=1.11 \mathrm{~L}$, and the ratio of the electron drift velocity to the electron thermal velocity is $\mathrm{v}_{z e} / \mathrm{v}_{t e}=0.16, m_{i} / m_{e}=100$, and $\omega_{p e} / \omega_{c e}=2$. These parameters mean that most of the ions are unmagnetized, and the collisionless tearing mode is stable. ${ }^{11}$ We carry out two kinds of simulation runs for $E_{0}=0$ and $E_{0}=-0.02 B_{0}$ to examine the dependence of simulation results on the spatial grid number. It is found that qualitatively similar results are obtained from each run, but an influence of boundary conditions becomes visible by changing the grid number along the $y$-axis for no driving field, while it becomes visible by changing the grid number along the $x$-axis for a finite driving field. We discuss the simulation results for case A $\left(E_{0}=0\right)$ in Sec. III and those for case $\mathrm{C}\left(E_{0}=-0.02 B_{0}\right)$ in Sec. IV.
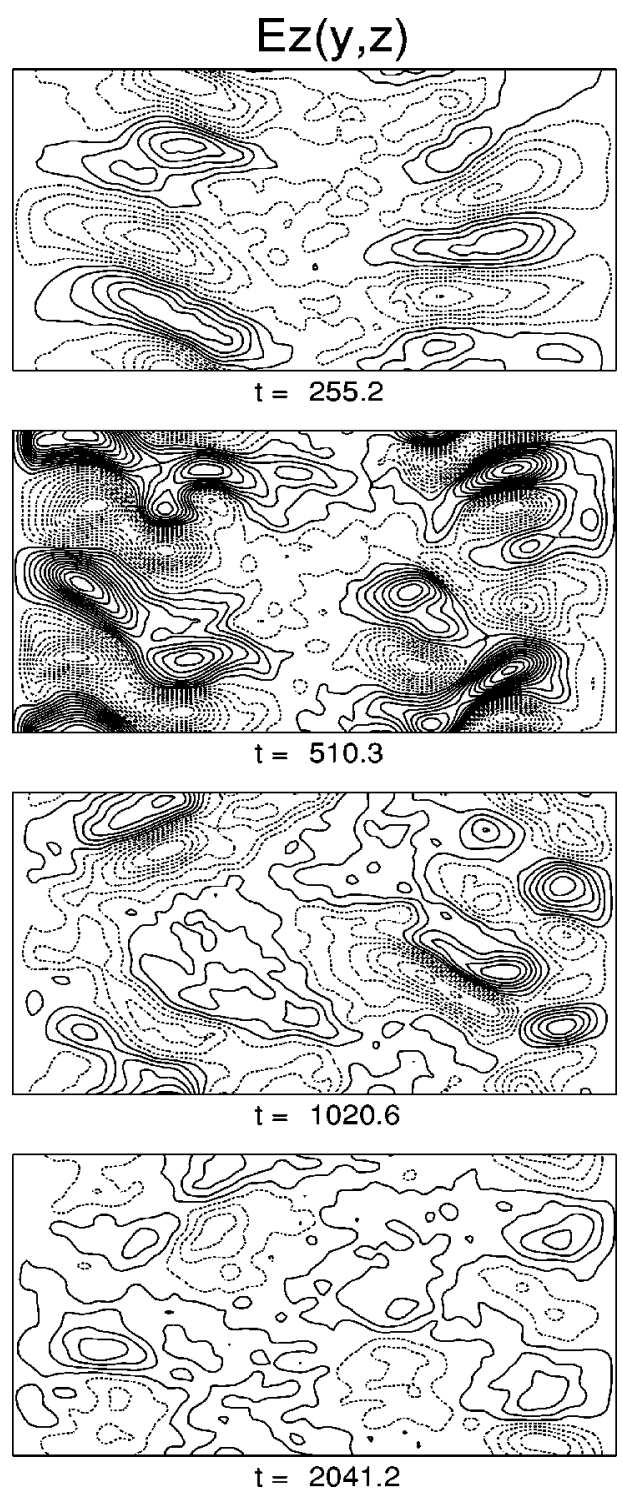

FIG. 2. Contour plots of an electric field $E_{z}$ in the $(y, z)$ plane at four different time periods for $E_{0}=0$ where the top, second, third, and bottom panels stand for the profiles at $\omega_{c e} t=255,510,1021$, and 2041, respectively.

\section{GENERATION OF ANOMALOUS RESISTIVITY}

Two-dimensional particle simulation ${ }^{20}$ has revealed that two kinds of plasma instabilities grow in the current layer. The LHDI $^{13,18,21,22}$ grows in the periphery of the current layer in the early phase, while the $\mathrm{DKI}^{23,24,26}$ is excited near the neutral sheet after the saturation of the LHDI. In this section we examine how these plasma instabilities evolve in three-dimensional space in the absence of an external driving flow $\left(E_{0}=0\right)$. Figures 2 and 3 show (i) the contour plots of an electric field $E_{z}$ and (ii) those of a magnetic field $B_{x}$ in the $(y, z)$ plane at four different time periods for case A where the top, second, third, and bottom panels stand for the profiles at $\omega_{c e} t=255,510,1021$, and 2041, respectively. The neutral sheet is located along the mid-vertical line. The fluctuation of $E_{z}$ with a relatively large wave-number grows in the periphery in the early temporal phase (second panel in Fig. 2). It is confirmed that the fluctuation of $E_{z}$ is dominated by an 

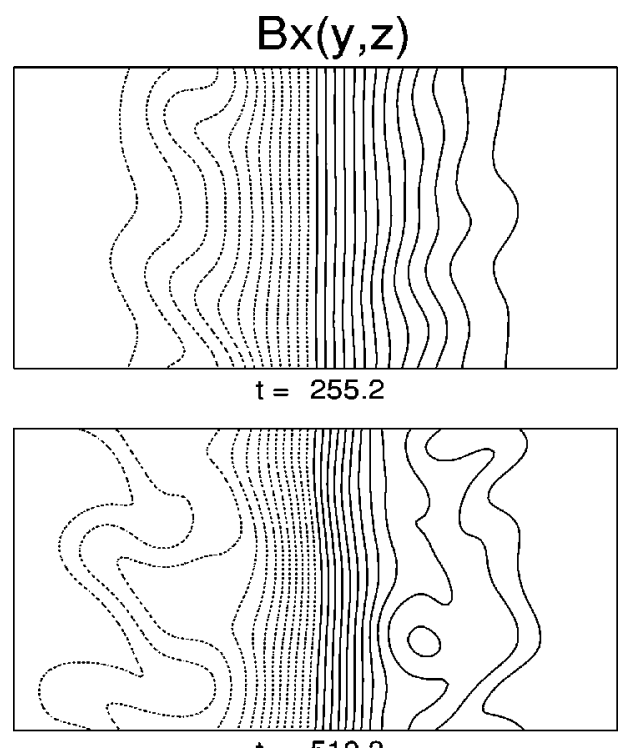

$\mathrm{t}=\mathbf{5 1 0 . 3}$
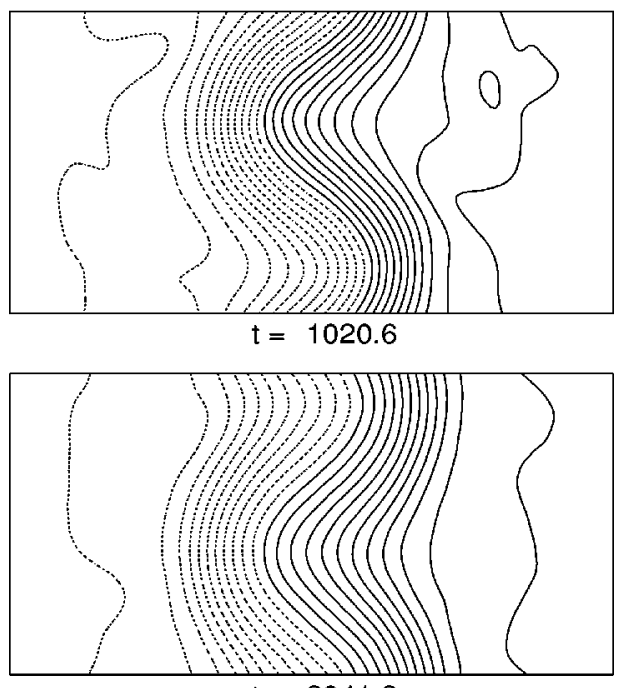

$t=2041.2$

FIG. 3. Contour plots of a magnetic field $B_{x}$ in the $(y, z)$ plane at four different time periods for the same case as Fig. 2.

electrostatic mode. After the saturation of this mode, the kink-like deformation of the current sheet becomes visible (third panel in Fig. 3). This mode pattern is kept for a long period while drifting in the ion drift direction with a phase velocity a little smaller than the ion diamagnetic drift velocity $\left(\omega / \mathbf{k} \cdot \mathbf{v}_{\mathrm{di}} \approx 0.7\right)$. These results are in good agreement with those for two-dimensional simulation. ${ }^{20}$ This is due to the fact that these modes do not create a spatial structure along the $x$-axis in a linear phase. ${ }^{30}$

Let us expand the spatial dependence along the $z$-axis into the Fourier modes. Shown in Figs. 4 and 5 are the frequency spectrum of an electric field $E_{z}^{(n)}(\omega)$ in the periphery $\left(y=-0.5 y_{b}\right)$ and that of a magnetic field $B_{x}^{(n)}(\omega)$ at the neutral sheet $(y=0)$ where $n$ is the Fourier mode number along the $\mathrm{z}$-axis, and the modes are calculated at $x=0$. Several wave-number modes are observed to grow simultaneously in the periphery, while only the $n=1$ mode is predominantly excited at the neutral sheet. According to the linear analysis of the drift kink (DK) mode, ${ }^{26}$ the growth rate

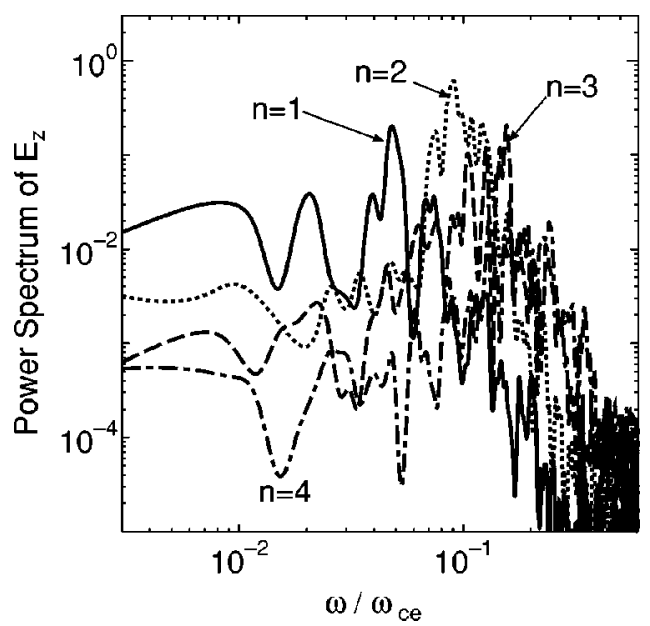

FIG. 4. Fourier spectrum of an electric field $E_{z}^{(n)}(\omega)$ in the periphery for the same case as Fig. 2 where the spatial profile along the $z$-axis at $(x, y)=(0$, $\left.-0.5 y_{b}\right)$ is expanded into the Fourier modes, and the solid, dotted, dashed, and dot-dashed lines stand for the frequency spectrum of the $n=1,2,3$, and 4 modes.

has a peak around $k_{z} L=1$ and the growth of kink mode is strongly suppressed for $k_{z} L \gg 1$. Because $k_{z} L \approx 2 n$ in the present case, the electromagnetic (EM) mode observed near the neutral sheet is qualitatively in good agreement with the linear analysis of the DK mode. Figure 6 displays the dispersion relation of $E_{z}$ at $y=-0.5 y_{b}$ for the same case as Fig. 4 where open squares stand for the simulation result and the solid curve is the theoretical dispersion relation of the lower hybrid drift (LHD) mode. ${ }^{13,21,22}$ It is obvious from Fig. 6 that the electrostatic mode excited in the periphery of the current layer is the LHD mode.

A time delay is observed between the excitation of two plasma instabilities. In order to clarify the physical meaning of the delay, we examine the temporal evolution of dominant Fourier modes in the current layer in more detail. Figure 7 plots the temporal evolution of Fourier amplitudes of three modes where the solid, dotted, and dashed lines stand for the Fourier amplitudes of $B_{x}^{(n=1)}$ at $y=0, E_{z}^{(n=2)}$ at

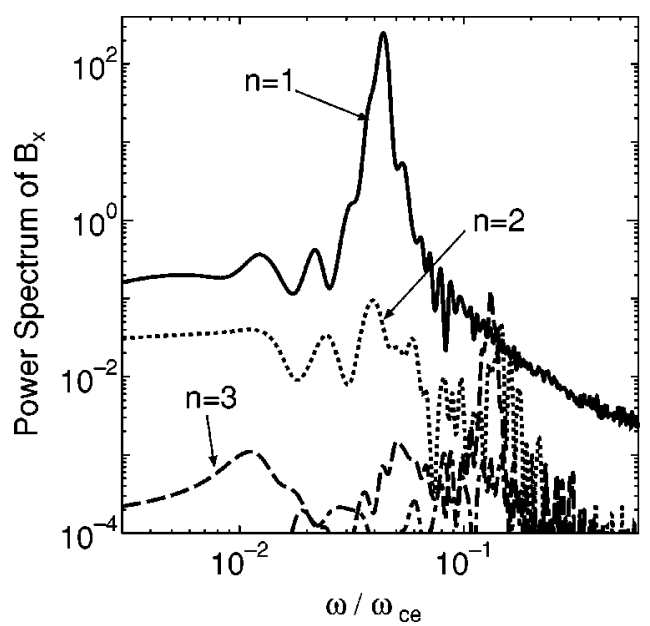

FIG. 5. Fourier spectrum of a magnetic field $B_{x}^{(n)}(\omega)$ at the neutral sheet $[(x, y)=(0,0)]$ for the same case as Fig. 2 . 


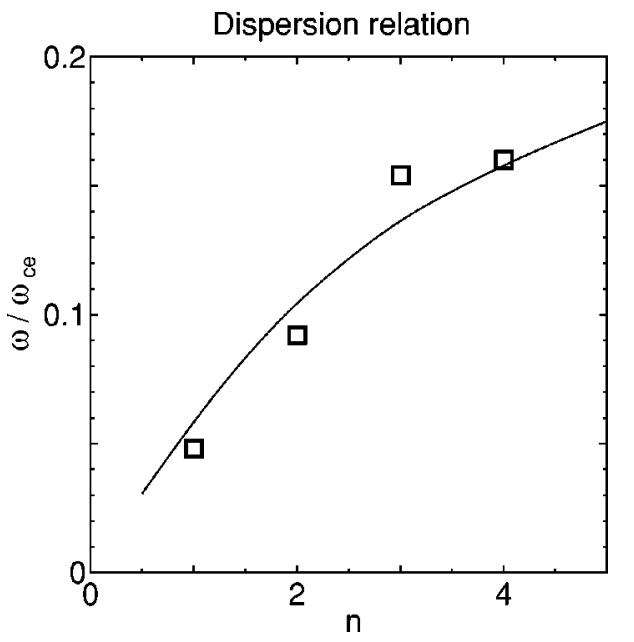

FIG. 6. Dispersion relation of $E_{z}$ at $y=-0.5 y_{b}$ for the same case as Fig. 4 where open squares stand for the simulation result and the solid curve is the theoretical dispersion relation of the LHD mode.

$y=-0.5 y_{b}$, and $E_{z}^{(n=0)}$ at $y=0$, respectively. It is insightful to discuss the mode behaviors by splitting time into five typical phases, i.e., (1) $0<\omega_{c e} t<190$, (2) $190<\omega_{c e} t<360$, (3) $360<\omega_{c e} t<840$, (4) $840<\omega_{c e} t<1350$, and (5) 1350 $<\omega_{c e} t<2000$. In phase 1 the LHD mode $E_{z}^{(n=2)}$ grows linearly, while both $B_{x}^{(n=1)}$ and $E_{z}^{(n=0)}$ remain at a noise level. That is, phase 1 corresponds to the linear phase of the LHDI. A sudden increase appears in the mode $B_{x}^{(n=1)}$ and the LHDI approaches the saturation level in Phase 2. The reconnection electric field $E_{z}^{(n=0)}$ starts to grow at the same rate as the DK mode $B_{x}^{(n=1)}$ in phase 3 . The reconnection electric field dissipates gradually and the DKI approaches its maximum value in phase 4 . Using the relaxation time $\tau_{\text {rel }}$ of the reconnection electric field in Phase 4, an anomalous resistivity induced by the DKI can be estimated as $\eta_{\text {DKI }}$ $\approx 4 \pi /\left(\omega_{p e}^{2} \tau_{\text {rel }}\right) \approx 8.8 \cdot 10^{-3} \omega_{p e}^{-1}$. The amplitude of the DK mode is almost constant and the field $E_{z}^{(n=0)}$ returns to the noise level in phase 5. Figure 7 suggests that the nonlinear saturation of the LHDI in phase 2 is deeply related to the

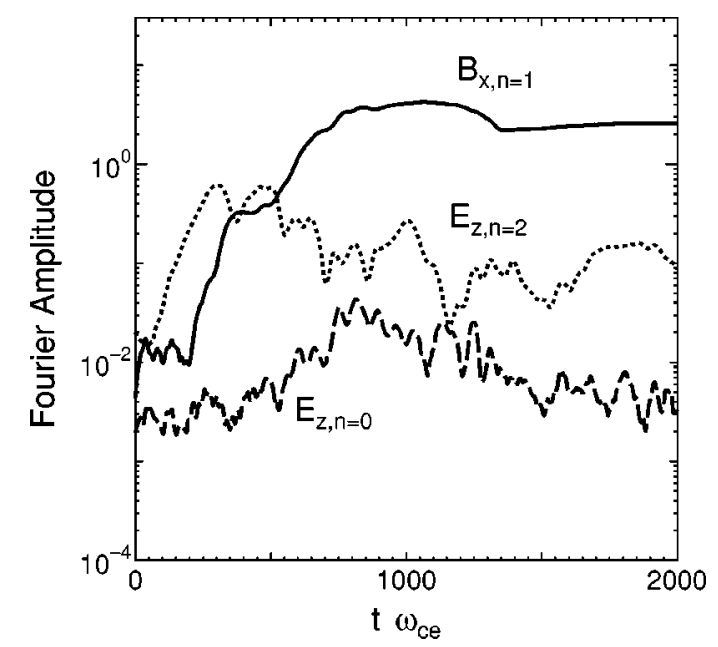

FIG. 7. Temporal evolutions of Fourier amplitudes of three modes for the same case as Fig. 2.

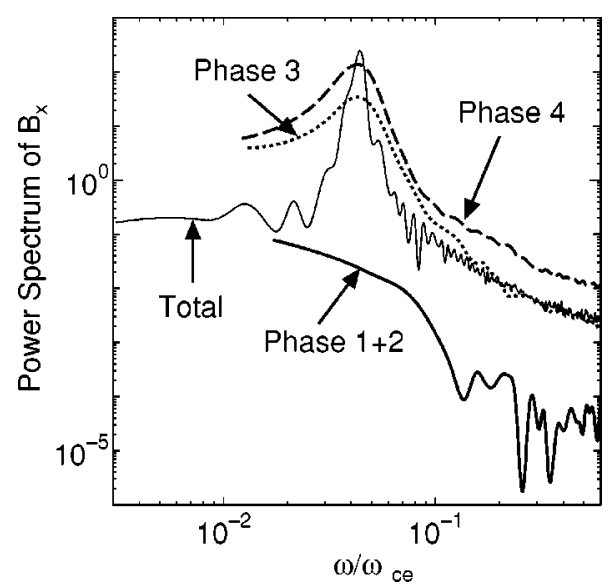

FIG. 8. Power spectrum of $B_{x}^{(n=1)}(\omega)$ for four time intervals, i.e., 1 $<\omega_{c e} t<360$ (solid), $360<\omega_{c e} t<840$ (dotted), $840<\omega_{c e} t<1350$ (dashed), and $1<\omega_{c e} t<2000$ (thin) for the same case as Fig. 2.

excitation of the DKI. Power spectrum of $B_{x}^{(n=1)}(\omega)$ is plotted in Fig. 8 for four time intervals, i.e., $1<\omega_{c e} t<360$ (solid), $\quad 360<\omega_{c e} t<840 \quad$ (dotted), $\quad 840<\omega_{c e} t<1350$ (dashed), and $1<\omega_{c e} t<2000$ (thin). A peak of the thin curve corresponds to the DK mode, the frequency of which is equal to $\omega=0.043 \omega_{c e}$. We cannot find any peak in the spectrum for phases 1 and 2 (solid). This means that the DK mode is stable in phases 1 and 2, and the sudden increase in the mode $B_{x}^{(n=1)}$ does not correspond to the excitation of the DK mode. The spectral curves indicate that the DKI is triggered in phase 3.

What happens in phase 2? Figure 9 illustrates the contour plots of the mode amplitudes of $B_{x}^{(n=1)}$ (top) and $E_{z}^{(n=2)}$ (second), the absolute values of the magnetic field (third), and the current density (bottom) at $x=0$ in the $(t, y)$ plane where the vertical and horizontal axes stand for the space $y$ and the time $t$ in a unit of $\omega_{c e}^{-1}$, respectively. One can see also in Fig. 9 that the LHDI grows in the periphery in early phase (second) and the DKI is triggered near the neutral sheet after the LHDI is nonlinearly saturated (top), as was seen in Fig. 2 and Fig. 3. It is worthy to note that an interesting phenomenon appears in the current density profile as a result of the nonlinear evolution of the LHD mode in phase 2 (bottom). The current profile is flattened in the periphery where the LHD mode is excited, while the current peaking takes place in the central region. In other words, the particle momentum is transferred from the periphery to the central region through the action of the electrostatic field excited by the LHDI. ${ }^{35}$ The compression by the LHD mode is not mirror-symmetric around the neutral sheet because the LHD modes above the sheet is independent of those below the sheet. The compression deforms the current sheet in the asymmetric way and triggers the DKI in the vicinity of the neutral sheet. Thus, the asymmetric compression by the LHD mode leads to the sudden increase in the mode $B_{x}^{(n=1)}$ in phase 2 (see Fig. 7).

The current layer becomes thinner than the initial profile as a result of the compression by the LHD mode. The temporal evolution of five spatial scales are plotted in Fig. 10 where the solid, dashed, dotted, dot-dashed, and dot-dot- 


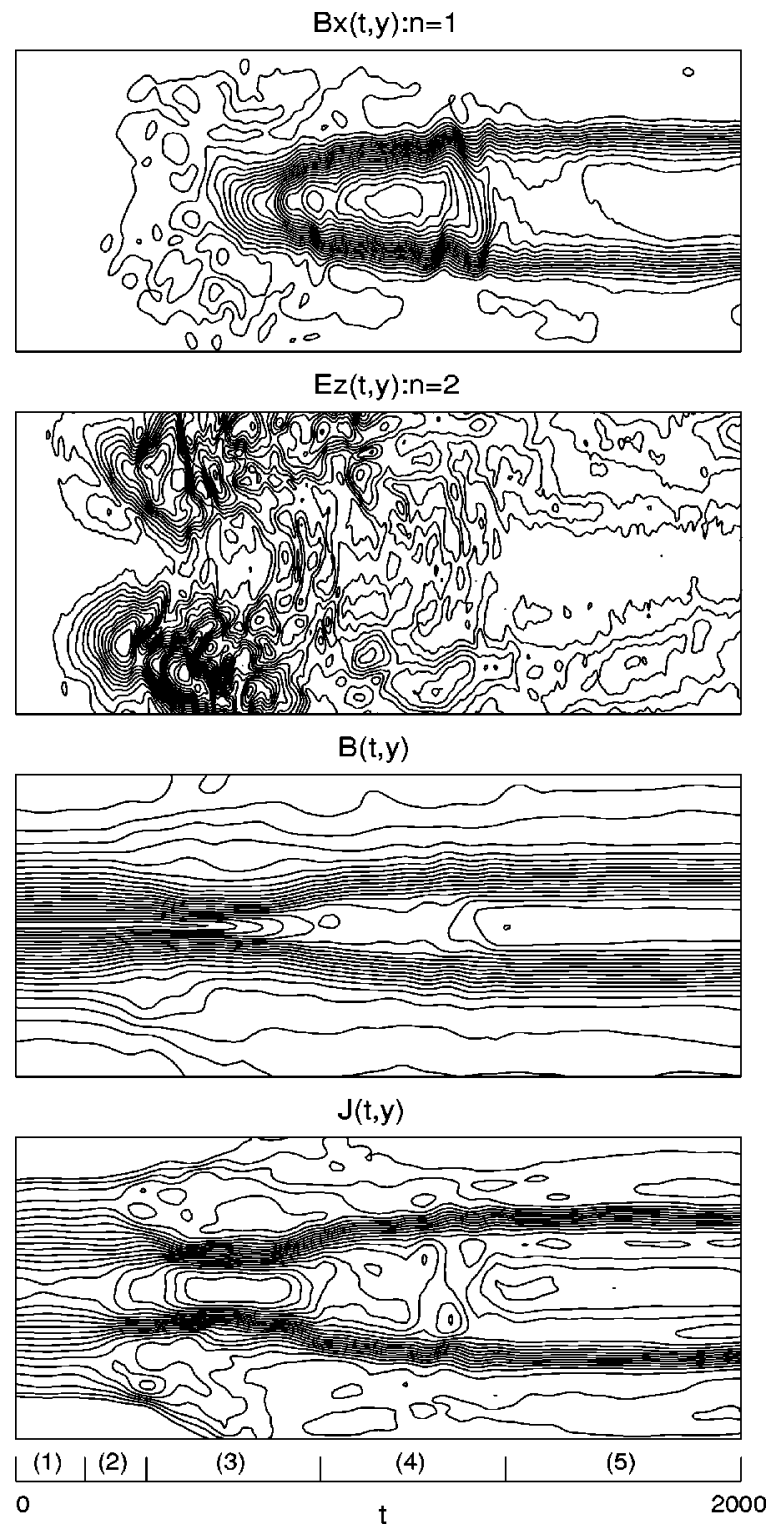

FIG. 9. Contour plots of the $n=1$ mode of the magnetic field $B_{x}$ (top), the $n=2$ mode of the electric field $E_{z}$ (second), the absolute values of the magnetic field (third), and the current density (bottom) at $x=0$ in the $(t, y)$ plane where the vertical and horizontal axes stand for the space $y$ and the time $t$ in a unit of $\omega_{c e}^{-1}$, respectively.

dashed lines stand for the half-width of current density $d_{j z}$, ion Larmor radius $\rho_{i}$, the electron skin depth $c / \omega_{p e}$, the ion skin depth $c / \omega_{p i}$, and the average amplitude of meandering electron orbit $l_{\mathrm{me}}$. The half-width of the current layer is nearly equal to the ion Larmor radius in the initial profile. The half-width starts to decrease in phase 2 and reaches its minimum value in phase 3 , while the ion Larmor radius remains almost constant. The relation $d_{j z} \approx 0.7 \rho_{i}$ holds in phase 3. The width $d_{j z}$ increases and approaches $\rho_{i}$ again in phase 4 . The behavior of the DKI is strongly correlated with the ratio of the half-width $d_{j z}$ to the ion Larmor radius $\rho_{i}$. That is, the DKI is triggered by decreasing the width $d_{j z}$ below $\rho_{i}$ in phase 3 . The nonlinear evolution of the DK instability leads to the flattening of the current profile in the central region and the increase in $d_{j z}$. The DK instability is

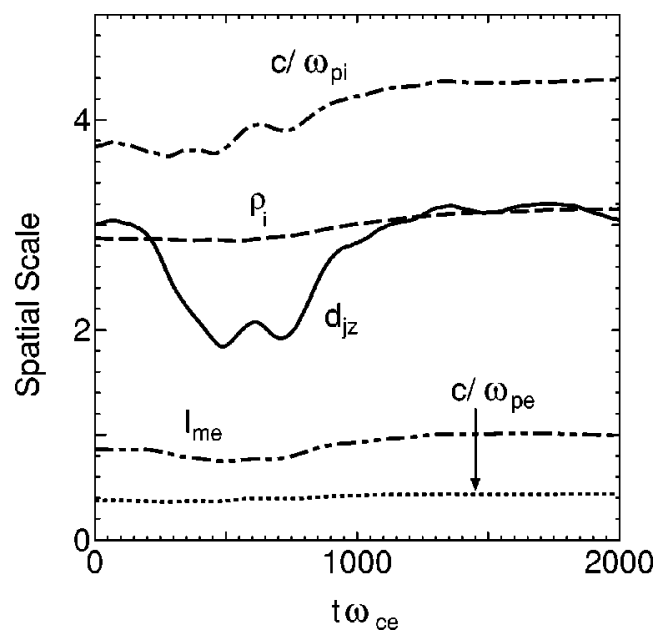

FIG. 10. Temporal evolution of five spatial scales for the same case as Fig. 2 where the solid, dashed, dotted, dot-dashed, and dot-dot-dashed lines stand for the half-width of current density $d_{j z}$, ion Larmor radius $\rho_{i}$, the electron skin depth $c / \omega_{p e}$, the ion skin depth $c / \omega_{p i}$, and the average amplitude of meandering electron orbit $l_{\mathrm{me}}$.

stabilized again in phase 5 when $d_{j z} \approx \rho_{i}$. These results are in good agreement with the linear kinetic theory ${ }^{23,24,26}$ and the reconnection experiments. ${ }^{3-5}$ The stabilization of the DKI by the ion magnetization effect was observed in the particle simulation of field-reversed configuration plasmas. ${ }^{36}$ On the other hand, the behavior of the DK instability is almost independent of the electron skin depth $c / \omega_{p e}$, the ion skin depth $c / \omega_{p i}$, and the electron meandering amplitude $l_{\text {me }}$. This result is quite different from the fluid models, ${ }^{8-10}$ in which a whistler mode is destabilized when the width decreases below the ion skin depth $c / \omega_{p i}$.

Because ions inside the current layer $\left(r \leqslant \rho_{i}\right)$ remain unmagnetized, the compression by the LHD mode acts only on electrons. Thus, the current layer is split into the ion and electron current layers. Figure 11 shows the temporal evolutions of average particle velocities $\left\langle\mathrm{v}_{e z}\right\rangle$ and $\left\langle\mathrm{v}_{i z}\right\rangle$, and ion thermal velocity $\mathrm{V}_{t i}$ at midpoint where the average velocity

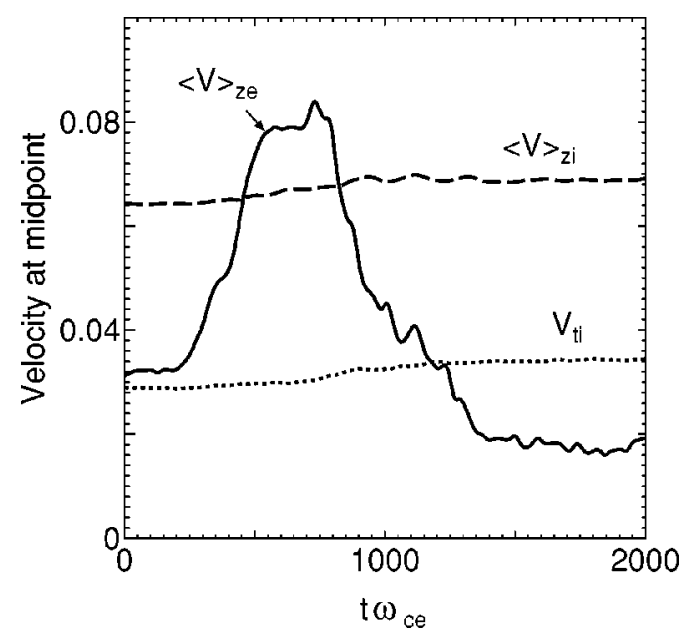

FIG. 11. Temporal evolutions of the $z$ component of the average electron velocity (solid), that of the average ion velocity (dashed), and ion thermal velocity (dotted) at midpoint for the same case as Fig. 2. 


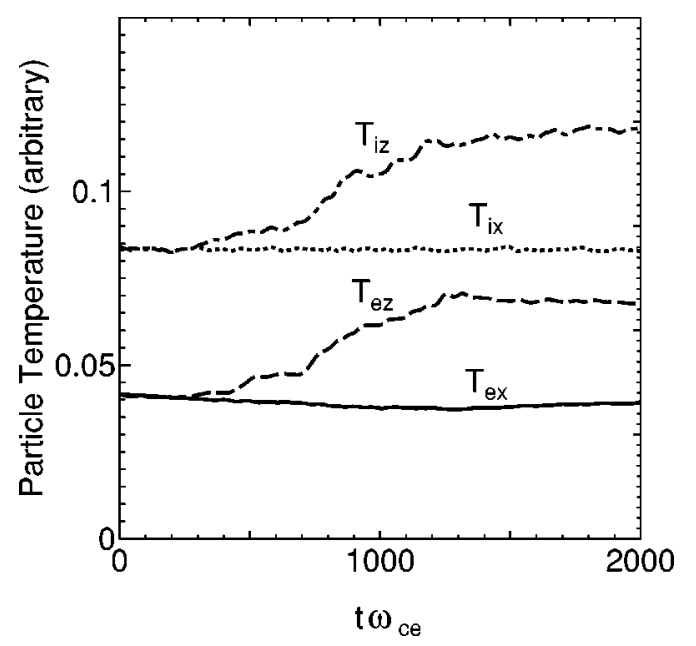

FIG. 12. Temporal evolutions of average particle temperature at the midpoint where the solid, dashed, dotted, and dot-dashed lines represent the $x$ component and $z$ component of the electron temperature and those of the ion temperature, respectively.

is defined in the same way as the current density in Eq. (8). The compression by the LHD mode leads to the increase in the average electron velocity $\left\langle\mathrm{v}_{e z}\right\rangle$ in the central region, but not in $\left\langle\mathrm{v}_{i z}\right\rangle$. The DKI plays a role in enlarging the current layer, while its growth rate decreases with increasing the width of the current layer. Consequently, the width of an electron current layer reaches its minimum value when the compression by the LHD mode is balanced with the growth of the DKI. It is interesting to note that the minimum width is determined by this balance condition, but not by the electron scales such as the electron skin depth or the electron meandering amplitude. This point is quite different from the simulation results in two dimensions. ${ }^{28,29}$

The excitation of two plasma instabilities gives rise to the anisotropic heating of plasmas in the current layer. Figure 12 shows the temporal evolutions of average particle temperature at the midpoint where the solid, dashed, dotted, and dot-dashed lines represent the $x$ component and $z$ component of the electron temperature and those of the ion temperature, respectively. The particle heating takes place in the perpendicular direction to an equilibrium magnetic field through the action of the DKI. We plot the spatiotemporal structure of the particle temperatures in Fig. 13 where the top, second, third, and bottom panels represent the $x$ component of the electron temperature $T_{e x}$, that of the ion temperature $T_{i x}$, the perpendicular component of the electron temperature $T_{e p}$, and that of the ion temperature $T_{i p}$, respectively. It is obviously shown in Fig. 13 that the electron temperature is heated up in the perpendicular direction inside the current layer in accordance with the excitation of the DKI. We can also see that the same phenomenon takes place in the ion temperature, although the profile is ambiguous due to the finiteness of the ion Larmor radius.

\section{EFFECT OF AN EXTERNAL DRIVING FIELD}

The reconnection electric field dissipates gradually in phase 4 and returns to the noise level in phase 5 , as was seen in Fig. 7. In other words, collisionless reconnection is termi-

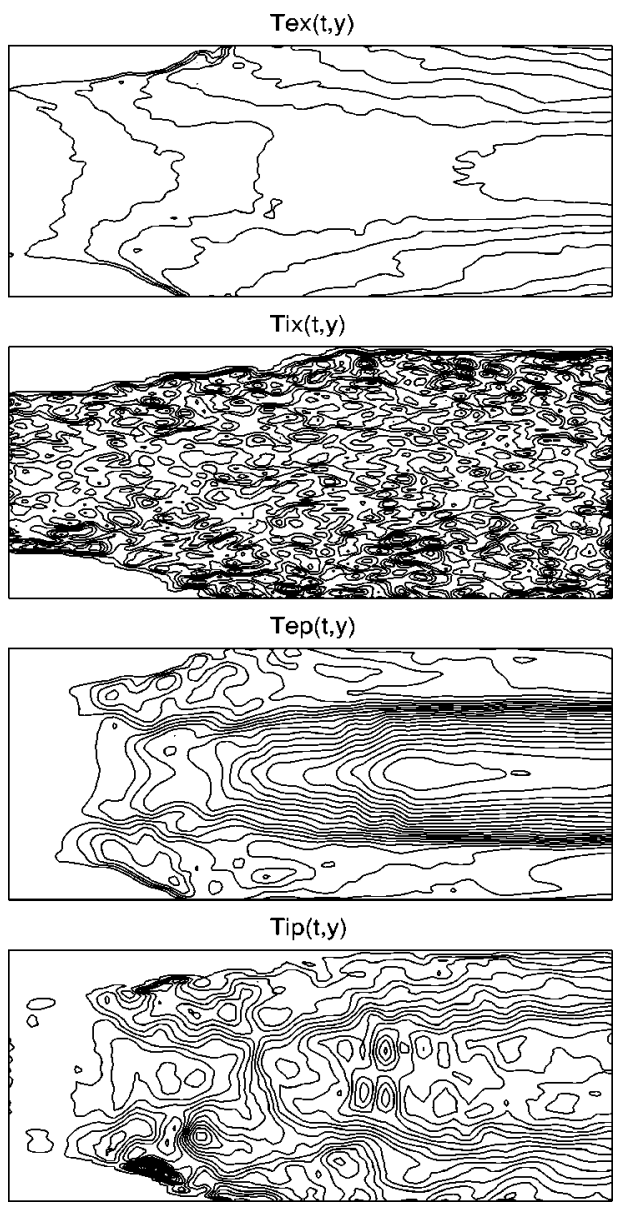

FIG. 13. Spatiotemporal structure of the particle temperatures in the $(t, y)$ plane where the top, second, third, and bottom panels represent the $x$ component of the electron temperature $T_{e x}$, that of the ion temperature $T_{i x}$, the perpendicular component of the electron temperature $T_{e p}$, and that of the ion temperature $T_{i p}$, respectively.

nated for a relatively short period in the absence of an external driving field. The magnetic flux is carried away from the periphery towards the central region through the action of the electrostatic field generated by the LHDI. However, the supply of a magnetic flux to the neutral sheet is slowed down and stopped in a short time because the LHDI is stabilized as a result of the current flattening. This phenomenon is also related to the fact that the total amount of magnetic flux in the periphery is limited. Thus, the magnetic flux supply from the exterior region is needed for the continuation of collisionless reconnection. According to the two-dimensional simulation in the presence of an external driving flow, ${ }^{28,29}$ the convective electric field can penetrate into the ion current layer after the current layer is compressed as thin as the ion kinetic scale by the convergent plasma flow. ${ }^{37}$ The Poynting flux moves towards the neutral sheet while creating the electron current layer inside the ion current layer. As soon as the electric field reaches the neutral sheet, magnetic reconnection is triggered and the topology of magnetic field lines is changed.

Let us examine how two plasma instabilities, the LHDI and the DKI, evolve in three dimensions in the presence of an external driving field. Figure 14 shows the spatiotemporal 


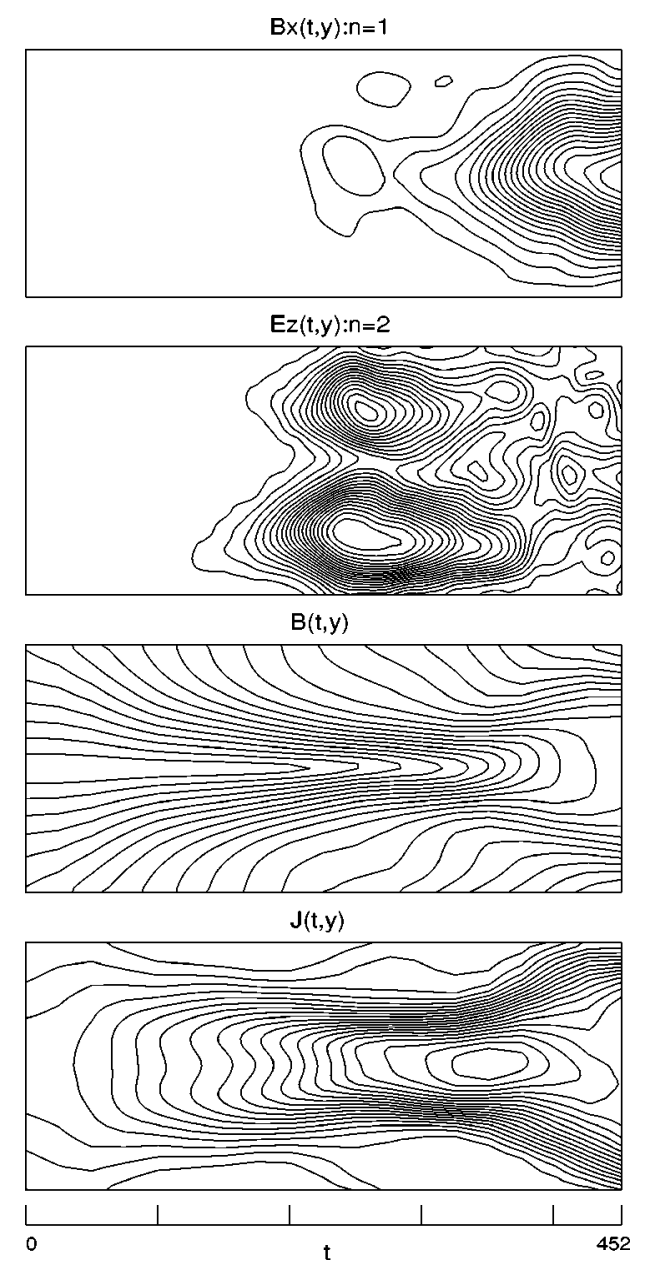

FIG. 14. Contour plots of the $n=1$ mode of the magnetic field $B_{x}$ (top), the $n=2$ mode of the electric field $E_{z}$ (second), the absolute values of the magnetic field (third), and the current density (bottom) at $x=0$ in the $(t, y)$ plane for case $\mathrm{C}$ where the vertical and horizontal axes stand for the space $y$ and the time $t$ in a unit of $\omega_{c e}^{-1}$, respectively.

structure of the $n=1$ mode of the magnetic field $B_{x}$ (top), the $n=2$ mode of the electric field $E_{z}$ (second), the absolute values of the magnetic field (third), and the current density (bottom) at $x=0$ in the $(t, y)$ plane for case $\mathrm{C}$ where the driving electric field is $E_{0}=-0.02 B_{0}$. One can see in Fig. 14 that the behaviors of the two instabilities are very similar to those for no driving field. That is, the LHDI is excited in the periphery in the intermediate temporal phase while the DKI is triggered near the neutral sheet in the late phase after the nonlinear deformation of the current layer by the LHDI. It is interesting to note that, in contrast to no driving field case, the LHD modes are forced to penetrate into the high beta central region by the external driving field and it keeps a finite amplitude even in the late phase. The temporal evolution of the four plasma modes are plotted in Fig. 15 for case $\mathrm{C}$ where the solid, dotted, dashed, dot-dashed lines stand for the Fourier amplitudes of the modes $B_{x}^{(n=1)}$ at $y$ $=0, B_{y}^{(m=1)}$ at $y=0, E_{z}^{(n=0)}$ at $y=0$, and $E_{z}^{(n=2)}$ at $y=$ $-0.5 y_{b}$, and $m$ is the Fourier mode number along the $x$-axis. Because most of the ions inside the current layer are unmagnetized in the present case, the electric field or the Poynting flux added at the input boundary penetrates into the current

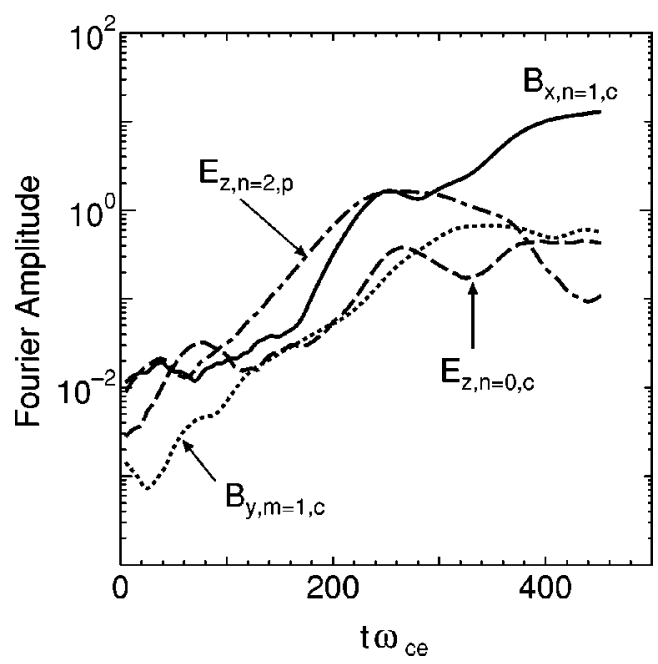

FIG. 15. Temporal evolutions of Fourier amplitudes of four modes for case $\mathrm{C}$.

layer while compressing mainly the electron profile. When the Poynting flux reaches the neutral sheet, collisionless reconnection is triggered and the $y$ component of the magnetic field is generated. The reconnection electric field starts to grow in the transit time for electrons to drift over the halflength of the simulation box along the $y$ direction $\left(t_{d e}\right.$ $=y_{b} B_{0} / c E_{0} \approx 100 \omega_{c e}^{-1}$ ). The penetration speed of the Poynting flux has a spatial dependence because there exist the Fourier modes of the electric field excited by the LHDI in the periphery. The asymmetric penetration gives rise to the rapid increase in the mode $B_{x}^{(n=1)}$ at the neutral sheet during the periods of $160<\omega_{c e} t<240$. It should be pointed out that magnetic reconnection is triggered by the convective electric field before the excitation of the DKI $\left(t>280 \omega_{c e}^{-1}\right)$.

Perspective views of the current sheet at three different periods are shown in Fig. 16 for case $\mathrm{C}$ where the current sheet is defined by the condition $B_{x}=0$, red and blue colors stand for the positive and negative values of magnetic field $B_{y}$. The initial straight current sheet is deformed into the $n$ $=1$ mode structure in the $z$ direction in the early phase (middle). The reconnection point appears almost along the midline $(x=0)$ in the current sheet (see green color). This is related to the fact that the applied electric field becomes maximum at the midline $(x=0)$ in the input boundary surface [see Eq. (13)]. The reconnected magnetic flux or the field $B_{y}$ is piled up in the downstream as time goes on. It is interesting to note that reconnection rate becomes maximum at the $z$ location where the DKI has a maximum amplitude, and thus the reconnected flux forms a two-dimensional spatial structure in the current sheet (bottom).

Because the convergent plasma flow supplied through the boundary compresses the current layer, the physical parameters such as the ion Larmor radius become a function of time. The temporal evolution of four spatial scales is shown in Fig. 17 where the solid, dashed, dotted, and dot-dashed lines stand for the half-width of current density $d_{j z}$, the ion Larmor radius $\rho_{i}$, the electron skin depth $c / \omega_{p e}$, and the average amplitude of meandering electron orbit $l_{\text {me }}$. The width $d_{j z}$ is comparable to ion Larmor radius $\rho_{i}$ in the initial 


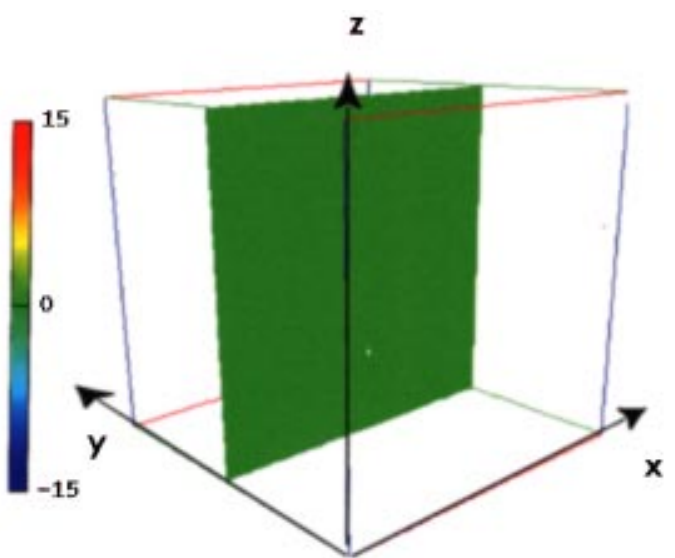

$\mathrm{t}=0$
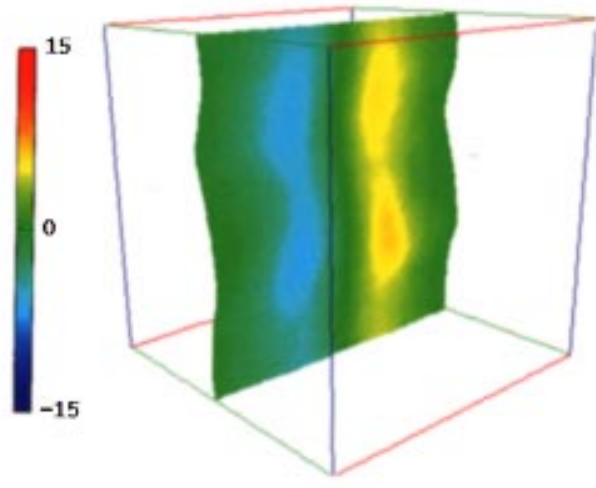

$\mathrm{t}=251$

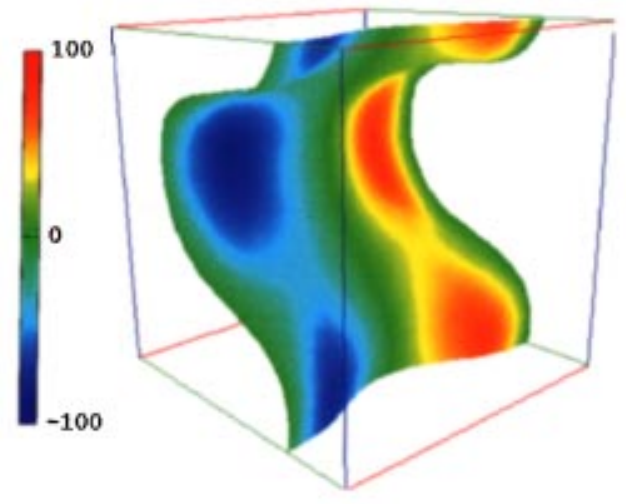

$\mathrm{t}=452$

FIG. 16. Perspective views of the current sheet at three different time periods for case $C$ where red and blue colors stand for the positive and negative values of magnetic field $B_{y}$.

profile. Both $d_{j z}$ and $\rho_{i}$ decrease with time, and $d_{j z}$ approaches one third of its initial value in the DKI growing phase, when the relation $d_{j z} \approx 0.5 \rho_{i}$ holds. The thinning of the current layer by the convergent plasma flow increases the growth rate of the DK mode, because the growth rate strongly depends on the ratio of the current layer width to the ion Larmor radius. ${ }^{23,26}$ Consequently, the DKI is triggered

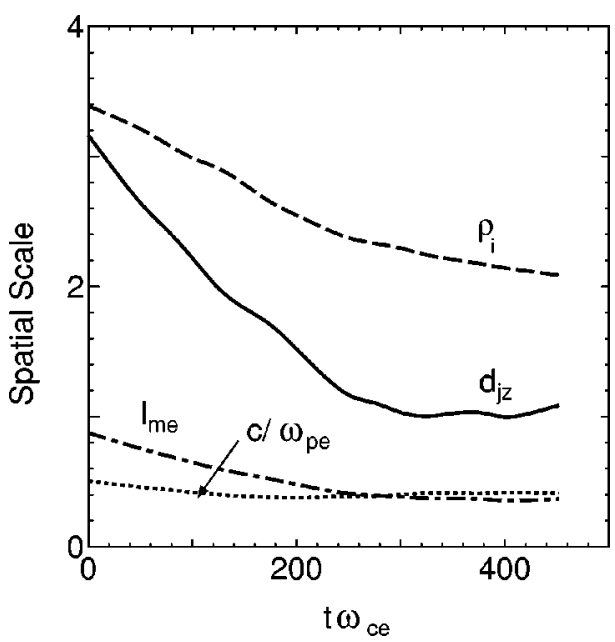

FIG. 17. Temporal evolution of four spatial scales for case $C$ where the solid, dashed, dotted, and dot-dashed lines stand for the half-width of current density $d_{j z}$, the ion Larmor radius $\rho_{i}$, the electron skin depth $c / \omega_{p e}$, and the average amplitude of meandering electron orbit $l_{\mathrm{me}}$.

earlier and grows with a larger rate compared with that for no driving flow.

Figure 18 shows the temporal evolution of the $z$ component of particle temperature at the midpoint for cases A and $\mathrm{C}$ where the solid, dotted, dashed, and dot-dashed lines represent the electron temperature and the ion temperature for case $\mathrm{C}$, and those for case A, respectively. As was seen in the two-dimensional studies, ${ }^{28,29}$ both the ions and the electrons are heated in the perpendicular direction to the equilibrium magnetic field through collisionless reconnection. Furthermore, it should be pointed out that the perpendicular component of ion temperature becomes much predominant over the other components and thus the ion velocity distribution becomes much anisotropic in the presence of an external driving field.

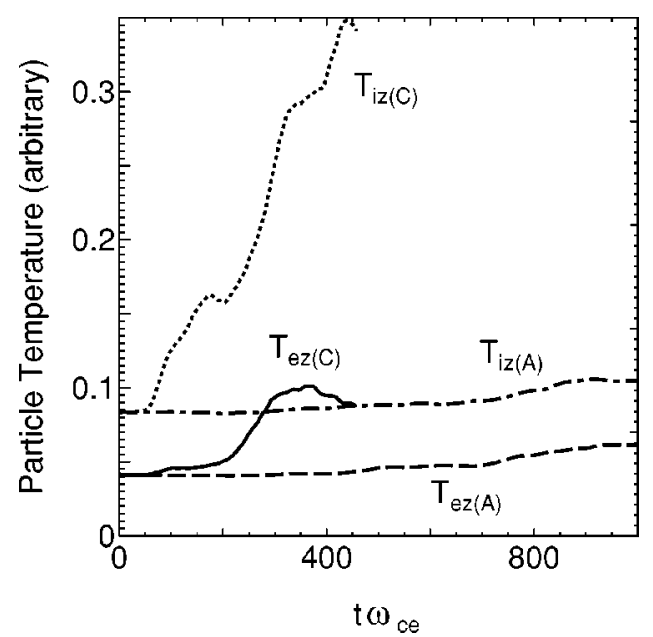

FIG. 18. Temporal evolution of the $z$ component of the particle temperature at the midpoint for cases $\mathrm{A}$ and $\mathrm{C}$ where the solid, dotted, dashed, and dot-dashed lines represent the electron temperature and the ion temperature for case $\mathrm{C}$, and those for case A, respectively. 


\section{SUMMARY AND DISCUSSIONS}

We have investigated the generation process of anomalous resistivity and the dynamical development of collisionless reconnection in three dimensions by means of a full particle simulation. For no external driving source, two different types of plasma instabilities are excited in the current layer. The lower hybrid drift instability (LHDI) is observed to grow in the periphery of the current layer in the early period. Nonlinear evolution of the LHDI leads to the current flattening in the periphery and the current peaking in the central region. The drift kink instability (DKI) is triggered at the neutral sheet after the half-width of the current layer decreases below that of the ion Larmor radius as a result of the nonlinear deformation of the current layer by the LHDI. An electric field grows at the neutral sheet in the same direction as the wavevector of the DKI in accordance with an excitation of the DKI, and thus collisionless reconnection is triggered by the DKI. An anomalous resistivity induced by the DKI can be estimated as $\eta_{\mathrm{DKI}} \approx 8.8 \times 10^{-3} \omega_{p e}^{-1}$. The DKI is also stabilized in a short period as a result of the current flattening in the absence of an external driving field.

When an external driving flow exists, the convective electric field penetrates into the current layer through the particle kinetic effect and collisionless reconnection is triggered by the convective electric field earlier than the DKI is excited. The LHD mode, which is excited in the periphery, is forced to penetrate into the high beta region by the convective electric field. This result is similar to the LHDI penetration model proposed by Winske. ${ }^{18}$ The reconnection rate becomes maximum at the point where the DKI has a maximum amplitude, and a two-dimensional structure of the reconnected magnetic flux is created in the current sheet. It is also found that the anisotropic ion distribution is formed through the anomalous ion heating by the DKI.

Finally, we make a comment on the relationship between the simulation results and the observations at the geomagnetic tail. Daughton ${ }^{17}$ has compared his linear analysis with the observation of substorm onset, ${ }^{38}$ and concluded that the DK instability cannot explain the onset because the growth rate is an order-of-magnitude weaker than the observed value $\left(\gamma / \omega_{c i} \approx 0.05\right)$. However, this disagreement is partly due to the fact that his analysis did not take into account the effect of nonlinear modification of the current layer by the LHDI. Our nonlinear simulation result shows that the growth rate is in the range of $0.35 \leqslant \gamma / \omega_{c i} \leqslant 0.96$ (see Table I). This value is an order-of-magnitude larger than the observed value. According to the linear analysis, ${ }^{17}$ the growth rate is reduced if the ion-to-electron mass ratio increases up to the real one. At the present, we cannot conclude whether the DK instability can explain the substorm onset because the nonlinear simulation is not carried out with the real mass ratio. This problem will be discussed elsewhere in the future.

\section{ACKNOWLEDGMENTS}

The simulation work was performed by employing the MISSION System (Grand Man-Machine Interactive System for Simulation) at the National Institute for Fusion Science. This work was supported in part by a Grant-in-Aid from the Ministry of Education, Science, and Culture in Japan (No. 10680468, No. 10044105, and No. 09480090).

${ }^{1}$ E. R. Priest, Solar Magnetohydrodynamics (Reidel, Dordrecht, 1982).

${ }^{2}$ A. Nishida, Geomagnetic Diagnostics of the Magnetosphere (SpringerVerlag, New York, 1978), p. 38.

${ }^{3}$ M. Yamada, Y. Ono, A. Hayakawa, M. Katsurai, and F. W. Perkins, Phys. Rev. Lett. 65, 721 (1990).

${ }^{4}$ Y. Ono, A. Morita, M. Katsurai, and M. Yamada, Phys. Fluids B 5, 691 (1993).

${ }^{5}$ Y. Ono, M. Inomoto, T. Okazaki, and Y. Ueda, Phys. Plasmas 4, 1953 (1997).

${ }^{6}$ J. A. Wesson, Nucl. Fusion 30, 2545 (1990).

${ }^{7}$ N. A. Krall and D. L. Book, Phys. Rev. Lett. 23, 574 (1969).

${ }^{8}$ J. F. Drake, R. G. Kleva, and M. E. Mandt, Phys. Rev. Lett. 73, 1251 (1994).

${ }^{9}$ D. Biskamp, E. Schwarz, and J. F. Drake, Phys. Rev. Lett. 75, 3850 (1995).

${ }^{10}$ D. Biskamp, E. Schwarz, and J. F. Drake, Phys. Plasmas 4, 1002 (1997).

${ }^{11}$ G. Laval, R. Pellat, and M. Vuillemin, Plasma Physics and Controlled Fusion Research (International Atomic Energy Agency, Vienna, 1966), Vol. II, p. 259

${ }^{12}$ B. Coppi, G. Laval, and R. Pellat, Phys. Rev. Lett. 16, 1207 (1966).

${ }^{13}$ N. A. Krall and P. C. Liewer, Phys. Rev. 4, 2094 (1971).

${ }^{14}$ K. Schinder, J. Geophys. Res. 79, 2803 (1974).

${ }^{15}$ J. D. Drake and Y. C. Lee, Phys. Rev. Lett. 39, 453 (1977).

${ }^{16}$ A. A. Galeev, Basic Plasma Physics II (North-Holland, New York, 1984), p. 305.

${ }^{17}$ W. Daughton, Phys. Plasmas 6, 1329 (1999).

${ }^{18}$ D. Winske, Phys. Fluids 24, 1069 (1981).

${ }^{19}$ M. Tanaka and T. Sato, J. Geophys. Res. 86, 5541 (1981).

${ }^{20}$ M. Ozaki, T. Sato, R. Horiuchi, and the Complex Simulation Group, Phys. Plasmas 3, 2265 (1996).

${ }^{21}$ R. C. Davidson and N. T. Gladd, Phys. Fluids 18, 1327 (1975).

${ }^{22}$ J. D. Huba, N. T. Gladd, and K. Papadopoulos, J. Geophys. Res. 83, 5217 (1978).

${ }^{23}$ K. Yamanaka, Phys. Scr. 17, 15 (1978).

${ }^{24}$ Z. Zhu and R. M. Winglee, J. Geophys. Res. 101, 4885 (1996).

${ }^{25}$ G. Lapenta and J. U. Brackbill, J. Geophys. Res. 102, 27099 (1997).

${ }^{26}$ W. Daughton, J. Geophys. Res. 103, 29429 (1998).

${ }^{27}$ M. Ottoviani and F. Porcelli, Phys. Rev. Lett. 71, 3802 (1993).

${ }^{28}$ R. Horiuchi and T. Sato, Phys. Plasmas 1, 3587 (1994).

${ }^{29}$ R. Horiuchi and T. Sato, Phys. Plasmas 4, 277 (1997).

${ }^{30}$ R. Horiuchi and T. Sato, Plasma Phys. Controlled Fusion 41, A477 (1999).

${ }^{31}$ V. A. Sergeev, D. G. Mitchell, C. T. Russell, and D. J. Williams, J. Geophys. Res. 98, 17345 (1993).

${ }^{32}$ T. Sato and T. Hayashi, Phys. Fluids 22, 1189 (1979).

${ }^{33}$ T. Sato, T. Hayashi, K. Watanabe, R., Horiuchi, M. Tanaka, N. Sawairi, and K. Kusano, Phys. Fluids B 4, 450 (1992).

${ }^{34}$ C. K. Birdsall and A. B. Langdon, Plasma Physics via Computer Simulation (McGraw-Hill, New York, 1985).

${ }^{35}$ W. Horton, Phys. Rev. Lett. 28, 1506 (1972).

${ }^{36}$ K. Nishimura, R. Horiuchi, and T. Sato, Phys. Plasmas 6, 3459 (1999).

${ }^{37}$ A. Fruchtman and K. Gomberoff, Phys. Fluids B 5, 2371 (1993).

${ }^{38}$ M. G. Henderson, G. D. Reeves, and R. D. Belian, Third International Conference on Substorms (European Space Agency, Versailles, France, 1996), p. 273. 